Res., Soc. Dev. 2019; 8(11):e308111474

ISSN 2525-3409 | DOI: http://dx.doi.org/10.33448/rsd-v8i11.1474

Competências para ler e escrever a língua inglesa presentes nos alunos de uma

\title{
Universidade Venezuelana
}

\section{Competencies for reading and writing the English language present in the students of a a venezuelan university}

Competencias para la lectura y escritura del idioma inglés presentes en los estudiantes de una universidad venezolana

Recebido: 03/08/2019 |. Revisado: 04/08/2019 |. Aceito: 07/08/2019 |. Publicado: 24/08/2019

Ysamar Sánchez Dávila

ORCID: https://orcid.org/0000-0003-1873-9607

Universidad Pedagógica Experimental Libertador, Venezuela

E-mail: ysamarschez@gmail.com

Jelly Katherine Lugo Bustillos

ORCID: https://orcid.org/0000-0002-0108-3771

Núcleo de Investigación Educativa Paraguaná, Venezuela

E-mail: jklb83@gmail.com

\section{Resumo}

O objetivo do estudo foi desvelar as habilidades de leitura e escrita dos alunos do Programa de Ensino de Língua Estrangeira em Língua Inglesa (ILE) da Universidad Nacional Experimental Francisco de Miranda (UNEFM) Municipal Carirubana, estado de Falcón, Venezuela. Ele foi enquadrado dentro do paradigma qualitativo, com um projeto de estudo de caso simples, porque ele trabalhou com um único cenário. Os informantes-chave foram seis (06) alunos do Programa IEF da UNEFM Municipalizada, do período I-2018. Para entender completamente o cenário do estudo e seu problema, utilizou-se a técnica de entrevista em profundidade. A análise das informações foi realizada com o apoio do software Atlas Ti 6.0, do qual os códigos foram extraídos e estruturados em redes semânticas, revelando que a falta de um amplo vocabulário, deficiências de coesão e coerência, aliada à desmotivação do Os alunos limitam significativamente a construção de conhecimento nesta área e o desenvolvimento de suas habilidades lingüísticas.

Palavras-chave: habilidades de leitura e escrita; língua estrangeira; inglês; coesão e coerência, vocabulário.

\section{Abstract}


The purpose of the study was to unveil the reading and writing skills of the students of the English Mention Foreign Language Education Program (ILE) of the UNEFM Municipal Carirubana, Falcón state, Venezuela. It was framed within the qualitative paradigm, with a simple case study design because it worked with a single scenario. The key informants were six (06) students of the IEF Program of the Municipalized UNEFM, from the I-2018 period. To thoroughly understand the study scenario and its problem, the in-depth interview technique was used. The analysis of the information was carried out with the support of the Atlas Ti 6.0 software, from which the codes were extracted and structured in semantic networks, revealing that the lack of a broad vocabulary, deficiencies in cohesion and coherence, coupled with the demotivation of the Students significantly limit the construction of knowledge in this area and the development of their language skills.

Keywords: reading and writing skills; foreign language; English; cohesion and coherence; vocabulary.

\section{Resumen}

El estudio tuvo como propósito Develar las competencias de lectura y escritura de los estudiantes del Programa de Educación en Lengua Extranjera Mención Inglés (ILE) de la UNEFM Municipalizada Carirubana, estado Falcón, Venezuela. Se enmarcó dentro del paradigma cualitativo, con un diseño de estudio de caso simple debido a que se trabajó con un solo escenario. Los informantes claves fueron seis (06) estudiantes del Programa de ILE de la UNEFM Municipalizada, del período I-2018. Para conocer a fondo el escenario de estudio y su problemática se utilizó la técnica de la entrevista en profundidad. El análisis de la información se realizó con el apoyo del software Atlas Ti 6.0, de donde se extrajeron los códigos y se estructuraron en redes semánticas, revelando que la carencia de un vocabulario amplio, deficiencias en la cohesión y coherencia, aunado a la desmotivación del estudiante limitan notablemente la construcción de conocimiento en esta área y el desarrollo de sus competencias lingüísticas.

Palabras clave: competencias de lectura y escritura; lengua extranjera; inglés; cohesión y coherencia; vocabulario.

\section{Introduction}


Como seres humanos vivimos expuestos a la necesidad de comunicarnos en un mundo con una cantidad innumerable de mensajes escritos por descifrar. De por sí realizamos de manera inconsciente el proceso de lectura de símbolos gráficos como parte de nuestro sentido elemental de comunicarnos, sin tomar en cuenta técnicas rigurosas para su comprensión, aunque reconocemos que el uso de técnicas es necesario para elevar la calidad y efectividad de la competencia comunicativa de los seres humanos (Español, 2016).

No obstante, para llevar a cabo ejercicios de lectura y escritura, se hace necesario indudablemente que quien lo realiza manifieste un alto grado de interés, concentración y disponibilidad a la comprensión. La clave para el éxito en este sentido es que con esas cualidades el sujeto se apoye en un análisis total del texto para profundizar sobre el mismo estableciendo sus propios criterios de reflexión.

La escritura específicamente es considerada por Oviedo (2015), como el arte de plasmar ideas o pensamientos de forma manuscrita, y como arte debe entenderse que también es un proceso. También es considerada como una manera de comunicarnos en la medida en que nos permite conocer las ideas y opiniones de otros. La escritura comparte con la lectura la necesidad de aplicación de técnicas para que fluya efectivamente entretanto al escribir en primer lugar requiere de una organización de ideas para luego ser plasmadas con coherencia y cohesión.

Ahora bien, para comprender una lectura en una lengua extranjera se requiere la construcción del significado del texto, proceso para el que el lector recurre a conocimientos previos o experiencias vividas relacionadas al tema. Al iniciar el proceso de lectura en una lengua extranjera se necesita de un poco más de dedicación ya que cuando el lector hace revisión de la literatura en una lengua extranjera encuentra obstáculos de mayor peso que le imposibilitan comprender y necesita de vocabulario perteneciente al idioma empleado de modo que pueda aplicar las estrategias asociadas a la lectura.

Ante este reto, Albarrán y otros (2016) plantean "además de las estrategias cognitivas el lector debe utilizar las meta cognitivas" que le permitirán planificar, monitorear, controlar y evaluar todo el proceso mental que interviene en la adquisición de estas competencias y que estas son aprendidas mediante la educación formal y eficiente al respecto.

Siendo este el contexto, Venezuela se ha visto en la necesidad de formar profesionales integrales con competencias para desenvolverse eficientemente en los diferentes ámbitos donde incursionen. Sin duda, el aprendizaje del idioma inglés como lengua extranjera es una herramienta para tal fin (Zeuch y Gregson, 2015). Por ello, desde todos los niveles educativos 
del país se propician los espacios de enseñanza y aprendizaje de esta segunda lengua. Las Casas de Educación Superior, entre las que destaca la Universidad Nacional Experimental Francisco de Miranda, tienen gran responsabilidad en la consecución de este propósito, ofreciendo a la comunidad en general la carrera de Educación en Lengua Extranjera Mención Inglés.

El Licenciado egresado de ésta formación especializada, debe cumplir con un perfil específico, el cual, según las políticas educativas del Estado, la política de formación docente y las políticas de docencia establecidas por el Área de Ciencias de la Educación de la UNEFM, es el de cumplir con los roles de facilitador, investigador, orientador, promotor social y administrador; además, tomando la enseñanza del idioma bajo un enfoque comunicativo. Moreno y Villafuerte (2016) plantean que la enseñanza bajo este enfoque debe considerar en primer lugar habilidades receptivas para inducir el reto de las habilidades de manera progresiva y acordes a las necesidades lingüísticas del estudiante

El enfoque comunicativo se relaciona directamente con las destrezas receptivas (en especial la de escuchar) y no intenta entrenar la producción oral; específicamente la fluidez oral que se espera que emerja de forma natural y gradualmente. Conforme se vayan mejorando las destrezas receptivas el estudiante finalmente alcanzará la fluidez y precisión al hablar.

En este sentido, las exigencias del Sistema Educativo en función del profesional egresado en el área de la enseñanza del inglés como lengua extranjera es que éste sea gerente e investigue en estrategias metodológicas, recursos y evaluación pero que a la vez comprenda la utilidad comunicativa del idioma, dominando destrezas para lograr aprendizajes significativos referentes al idioma. Es así que, en la presente investigación nos planteamos Develar la Competencias que tienen los estudiantes del Programa de Educación en Lengua Extranjera Mención Inglés (ILE) de la UNEFM Municipalizada Carirubana en relación a la lectura y escritura del idioma Inglés a fin de valorar aquellas fortalezas y debilidades que se presentan en la enseñanza y aprendizaje de la misma dentro del programa universitario de esta casa de estudios, lo cual influirá notoriamente en la toma de decisiones posterior para la implementación de estrategias de mejora.

\section{Lectura y Escritura en Lengua Extranjera}

Una de las metas que persigue el sistema educativo formal, específicamente, la Educación Universitaria es el logro de un proceso comunicativo eficaz y adecuado que permita alcanzar los objetivos pedagógicos establecidos por el docente y promueva el 
desarrollo exitoso del proceso de aprendizaje del estudiante. Es por ello que la comunicación, a través de sus diferentes manifestaciones (oral, escrita, gestual, etc.), constituye una herramienta de gran utilidad en el aula de clases (Ramírez y Acosta, 2015).

Para comunicarse efectivamente, tanto el docente como el estudiante deben dominar las cuatro destrezas lingüísticas (hablar, escuchar, escribir y leer), con la finalidad de que pueda aprender el inglés bajo un enfoque comunicativo, puesto que el objetivo del mismo, de acuerdo con Moreno y Villafuerte (2016) se basa en enseñar las funciones comunicativas que se derivan de las necesidades lingüísticas del alumno. La enseñanza de los aspectos formales y estructurales del lenguaje constituye aún un aspecto importante de la instrucción en las lenguas extranjeras, pero no al considerarlas como un fin, pues en sí no refleja la intención o el propósito comunicativo del hablante, sino más bien como un medio para alcanzar determinado fin: desarrollar la competencia comunicativa del sujeto hablante.

Brown (2006) define la competencia comunicativa como "la habilidad de usar el lenguaje apropiadamente en la interacción social, así como también la habilidad de comunicarse apropiadamente en forma oral y escrita".

Siendo así, la competencia para escribir en forma comunicativa requiere de la integración sistematizada de los componentes estructurales y funcionales del lenguaje, es decir, de la habilidad de usar elementos lingüísticos, a saber, sintaxis, morfología, semántica entre otros, combinados con los aspectos de índole pragmático, los cuales permiten expresar las ideas e información de forma apropiada y en un contexto específico. Para alcanzar este objetivo es necesario que el escritor esté consciente de que escribir no consiste sólo en un vaciado de símbolos y letras en un papel, sino que se constituye en un proceso complejo que integra diversos y variados aspectos y que tiene como fin, al igual que la comunicación oral, la interacción social del escritor con su audiencia. Ante esto, Díaz y Hernández (2004) consideran que

La interacción en el proceso de escritura no se realiza cara a cara, sino que se exige por parte del escritor, una mayor colaboración y un mayor compromiso en la elaboración de los textos producidos, a fin de lograr una comunicación efectiva y completa (p.18).

Con base en todo lo expuesto hasta ahora, se plantea entonces la inquietud a abordar, la cual tiene que ver con las competencias de lectura y escritura presentes en los estudiantes del Programa de Inglés de la UNEFM Municipalizada Carirubana, la cual intenta comprender los procesos de una habilidad receptora y otra productora y todos los elementos que estas conllevan. 
Partiendo de las observaciones realizadas por la investigadora como docente del área en la Casa de Estudios precitada, en diferentes grupos existen debilidades en los procesos de lectura y escritura de textos en inglés sobre todo en los niveles avanzados del Programa de Pregrado. Con respecto a la lectura, son estudiantes que tienen dificultades para comprender textos en el idioma extranjero y al escribir demuestran errores de gramática, especialmente en lo que a nivel sintáctico se refiere, las formas cómo relacionan las palabras en una oración y el modo en que combinan las expresiones dentro de un discurso para dar sentido y coherencia a un texto. Pareciera que estos elementos tienen que ver con la formación de base, o quizás con las estrategias de enseñanza y aprendizaje a la que están expuestos en su formación académica. Sin duda que los rasgos evidenciados son una inquietud que bien merece ser estudiada, considerando también que los egresados del Programa deben poseer habilidades lingüísticas que le permitan el posterior ejercicio efectivo de sus funciones como formadores en el idioma extranjero.

\section{Methodology}

Como ya se ha dicho, el objetivo de esta investigación fue Develar la Competencias que tienen los estudiantes del Programa de Educación en Lengua Extranjera Mención Inglés (ILE) de la UNEFM Municipalizada Carirubana en relación a la lectura y escritura del idioma inglés.

El estudio se fundamentó en el paradigma cualitativo, apoyado en el método hermenéutico dialectico. El diseño respondió a un estudio de caso que para Yin (1994):
Es una investigación empírica que estudia un fenómeno contemporáneo dentro de su contexto de la vida real, especialmente cuando los límites entre el fenómeno y su contexto no son claramente evidentes. Una investigación de estudio de casos trata exitosamente con una situación técnicamente distintiva en la cual hay muchas más variables de interés que datos observacionales $\mathrm{y}$, como resultado, se basa en múltiples fuentes de evidencia, con datos que deben converger en un estilo de triangulación; además, se beneficia del desarrollo previo de proposiciones teóricas que guían la recolección y el análisis de datos (p.38)

Así pues, para analizar las competencias de lectura y escritura presentes en los estudiantes de ILE de la UNEFM Municipalizada Carirubana se utilizó la técnica de la entrevista en profundidad, apoyada con un guion semi-estructurado contentivo de 12 preguntas abiertas, los cuales estaban divididos en dos subtemas: procesos de lectura y 
procesos de escritura, ambos sostenían seis preguntas aplicadas a seis (06) estudiantes pertenecientes al Programa antes descrito, posterior a esto los datos recolectados del encuentro fueron transcritos y se procesaron a través del Software Atlas Ti 6.0 para la respectiva codificación y categorización de la información y construcción de redes semánticas que al final fueron interpretadas y sintetizadas para simplificar la información recabada.

\section{Los hallazgos: discusión y evaluación de la praxis}

El programa Atlas Ti en su versión 6.0 permitió codificar y categorizar la información de las preguntas abiertas arrojando sesenta y cinco (65) códigos interrelacionados, los cuales se agruparon en tres categorías: Proceso de Lectura, Proceso de Escritura y Necesidades, Gustos e Intereses, ésta última funge como categoría emergente. En este sentido, se realiza la interpretación de las categorías a través del uso de las redes semánticas lo cual facilita el análisis desde el punto de vista de la investigadora en relación a las competencias de lectura y escritura presentes en los estudiantes del Programa de ILE de la UNEFM Municipalizada Carirubana. En el Cuadro 1 se muestra la leyenda utilizada para relacionar los diferentes códigos extraídos de la entrevista en profundidad.

\section{Cuadro 1 - Codificación Abierta}

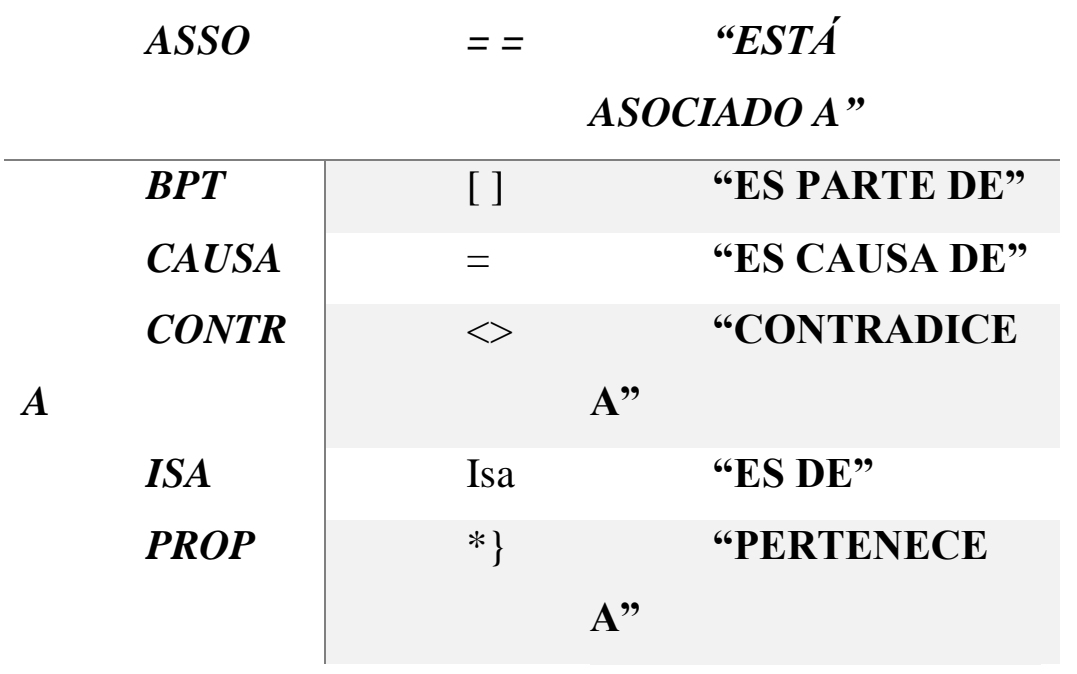

Fuente: Autores

Como se observa en el Cuadro 1, fueron cinco las relaciones que se establecieron entre los códigos emergentes de las entrevistas realizadas, la asignación de las mismas permitió sintetizar la información y estructurarla de manera comprensiva.

Asimismo, presentamos a continuación en el Cuadro 2, el listado de códigos que 
Res., Soc. Dev. 2019; 8(11):e308111474

ISSN 2525-3409 | DOI: http://dx.doi.org/10.33448/rsd-v8i11.1474

comprenden la Unidad Hermenéutica de Información, en total fueron sesenta y cinco (65) códigos emergentes.

\section{Cuadro. 2 - Lista de Códigos}

\section{Códigos}

\begin{tabular}{|c|c|}
\hline 1. & Acudir a algún profesor \\
\hline 2. & Agregar Información extra \\
\hline 3. & Ampliar Vocabulario \\
\hline 4. & Analizar el escrito en una lengua extranjera \\
\hline 5. & Angustia por el uso inadecuado de la gramática \\
\hline 6. & Atracción visual \\
\hline 7. & Buena capacidad para el análisis \\
\hline 8. & Buena fluidez \\
\hline 9. & Buena ortografía \\
\hline 10. & Buscar en libros \\
\hline 11. & Búsqueda de palabras clave \\
\hline 12. & Capacidad para realizar una lectura básica \\
\hline 13. & Coherencia en el escrito \\
\hline 14. & Dependiendo del estado de animo \\
\hline 15. & Dependiendo del tipo de texto \\
\hline 16. & Desconocimiento de la terminología léxico y sintaxis \\
\hline 17. & Desconocimiento de las conceptualizaciones de idea principal y secundaria \\
\hline 18. & Desconocimiento de la estrategias de comprensión \\
\hline 19. & Desconocimiento en la pronunciación de nuevas palabras \\
\hline 20. & Dificultad al comprender la idea principal de un texto complejo \\
\hline 21. & Dificultad para comprender vocabulario técnico \\
\hline 22. & Documentarse \\
\hline 23. & Elaborar borradores \\
\hline 24. & Errores de sintaxis \\
\hline 25 . & Escasez de conocimientos previos \\
\hline 26. & Escaso control y dirección del proceso lector \\
\hline 27. & Escritura clara \\
\hline 28. & Evitar errores gramaticales \\
\hline
\end{tabular}


ISSN 2525-3409 | DOI: http://dx.doi.org/10.33448/rsd-v8i11.1474

29. Exigencias de unidades curriculares

30. Gusta pero no fascina

31. Gusto por escribir

32. Identifica las ideas principales y secundarias

33. Identifica los tipos de textos

34. Interés

35. Lector pasivo

36. Lector regular

37. Lectura rápida

38. Manejo de vocabulario básico

39. Mantener contacto con la literatura inglesa

40. Mejorar vocabulario

41. Necesidad

42. No poseer un amplio vocabulario

43. Omisión de palabras

44. Párrafos necesarios

45. Placer de leer

46. Pobreza de vocabulario

47. Practicar el idioma

48. Problemas de memoria

49. Proceso de identificación de idea principales y secundarias lento

50. Rechazo a la escritura

51. Revisión de material archivado

52. Sentimientos y emociones

53. Solicitar ayuda a un experto

54. Ubicación de ideas al terminar la lectura

55. Ubicar palabras clave

56. Usar vocabulario que ya se tiene

57. Uso correcto de las pausas

58. Uso de gráficos

59. Uso de la internet

60. Uso del diccionario

61. Uso del subrayado 
62. Uso extenso de vocabulario

63. Uso incorrecto de la gramática

64. Uso incorrecto de los signos de puntuación

65. Verificar detalles

Fuente: autores

Los códigos expuestos en el Cuadro 2 expresan la síntesis de las unidades de información extraídas de las entrevistas, y responden a dos categorías estudiadas inicialmente: Procesos de Lectura y Procesos de Escritura, y a una tercera que resultó emergente en el discurso de los estudiantes y que para efectos de organización de información la hemos identificado con el nombre de Necesidades, Gustos e Intereses.

Atendiendo a las consideraciones anteriores se procede entonces a realizar el análisis interpretativo de cada red semántica arrojada.

\section{Categoría N$^{\circ} 01$ PROCESOS DE LECTURA}

Esta categoría representa todos los procesos y elementos que involucra la lectura. En esta red se generaron treinta (30) códigos los cuales se interrelacionaron entre sí (ver gráfico 1). Los estudiantes manifestaron que las razones por las que leen un texto en inglés son por interés, por exigencia de unidades curriculares, para ampliar su vocabulario, o para mantener contacto con la literatura inglesa, lo que significa practicar el idioma que a su vez guarda relación con la atracción visual, si el texto o libro no llama la atención no se lee, aunque si es un requisito exigido debe leerse por obligación. Mientras que unos se consideran lector pasivo o lector regular lo que repercute no sólo en mantener una buena fluidez en la lectura sino en identificar los tipos de textos debido a que se van familiarizando con la literatura, hay quienes tienen dificultad para comprender la idea principal en un texto complejo, lo que hace que el proceso de identificación de ideas principales y secundarias sea lento.

Así mismo ubican las ideas principales y secundarias al finalizar la lectura, no es que hacer esto esté mal, es que quizás deba releer el texto para ubicarlas, generalmente la localización de estos elementos fundamentales de la lectura se va haciendo a medida que se va leyendo.

Por otra parte, hay quienes, como estrategias, hacen uso del subrayado o uso de gráficos para obtener una mejor comprensión. Sin embargo, otros informantes relataron que poseen desconocimiento de las estrategias de comprensión, lo que hace que exista un escaso 
control y dirección del proceso lector. En relación a la búsqueda de ideas principales y secundarias algunos identifican las ideas principales y secundarias lo que da como resultado la buena capacidad para el análisis. Del mismo modo, refirieron que la lectura de párrafos necesarios se realiza dependiendo del tipo de texto, lo que lleva a pensar que se hace una lectura rápida, pero con el uso correcto de las pausas donde se haga una búsqueda de palabras clave que permitan la comprensión del contexto en el que se desarrolla el texto.

De igual manera, uno que otro informante relató que presentaba problemas de memoria para recordar palabras difíciles, lo que surgía como dificultad para comprender vocabulario técnico, lo que, a su vez, se relaciona con el desconocimiento en la pronunciación de nuevas palabras quizás no se trate de un problema de gran magnitud, pero por el simple hecho de no saber con exactitud lo que éste quiere decir la capacidad para realizar una lectura básica se convierte en nula.

Además de lo descrito anteriormente se pudo conocer que existe desconocimiento de la terminología léxico y sintaxis, así como el desconocimiento en las conceptualizaciones de ideas principales y secundarias lo que incide considerablemente de forma negativa en el proceso de comprensión del estudiante.

En el Cuadro 3 mostramos extractos de las respuestas expresadas en la entrevista a los estudiantes, las mismas dan cuenta de las unidades de análisis estudiadas.

\section{Cuadro 3.}

\section{Categoría. Procesos de Lectura}

\section{Informante Unidad de Análisis}

\footnotetext{
1 "primero que sea algo de mi interés, verdad y sobre todo la portada del libro, o de algo que me interese"

..."a veces me toca leerlo porque tengo que estudiar o por lo menos yo que estudio inglés generalmente estoy leyendo textos en inglés pues, primero para saber algo, segundo porque hay unas materias que requieren de lectura"

3 "Ehh bueno para mejorar mi vocabulario y ampliarlo"

"Bueno Depende del texto, a veces leo porque quiero averiguar información que solamente está disponible en el idioma inglés"

"Ehh Principalmente creo que por necesidad, soy de las personas que me gusta informarme y mucha de las cosas de mi interés están en ingles así que
} 
Res., Soc. Dev. 2019; 8(11):e308111474

ISSN 2525-3409 | DOI: http://dx.doi.org/10.33448/rsd-v8i11.1474

principalmente por eso y segundo también es una forma de practicar mi inglés y mejorar mi lenguaje"

"Porque mi carrera me lo exige, y bueno me gusta leer textos en inglés pero generalmente los leo porque me lo exige una unidad curricular en específico y bueno también porque así ganas vocabulario y además que es una habilidad que debes desarrollar en el programa"

Fuente: Informantes Clave.

De esta manera se evidencia cómo conciben los estudiantes los aspectos relacionados a la lectura en el idioma inglés. Para complementar tal perspectiva, presentamos en el Gráfico 1 la red semántica construida con el apoyo del Software Atlas Ti 6.0, donde se exponen los códigos y las relaciones resultantes para esta categoría de análisis.

Gráfico 1 - Procesos de Lectura.

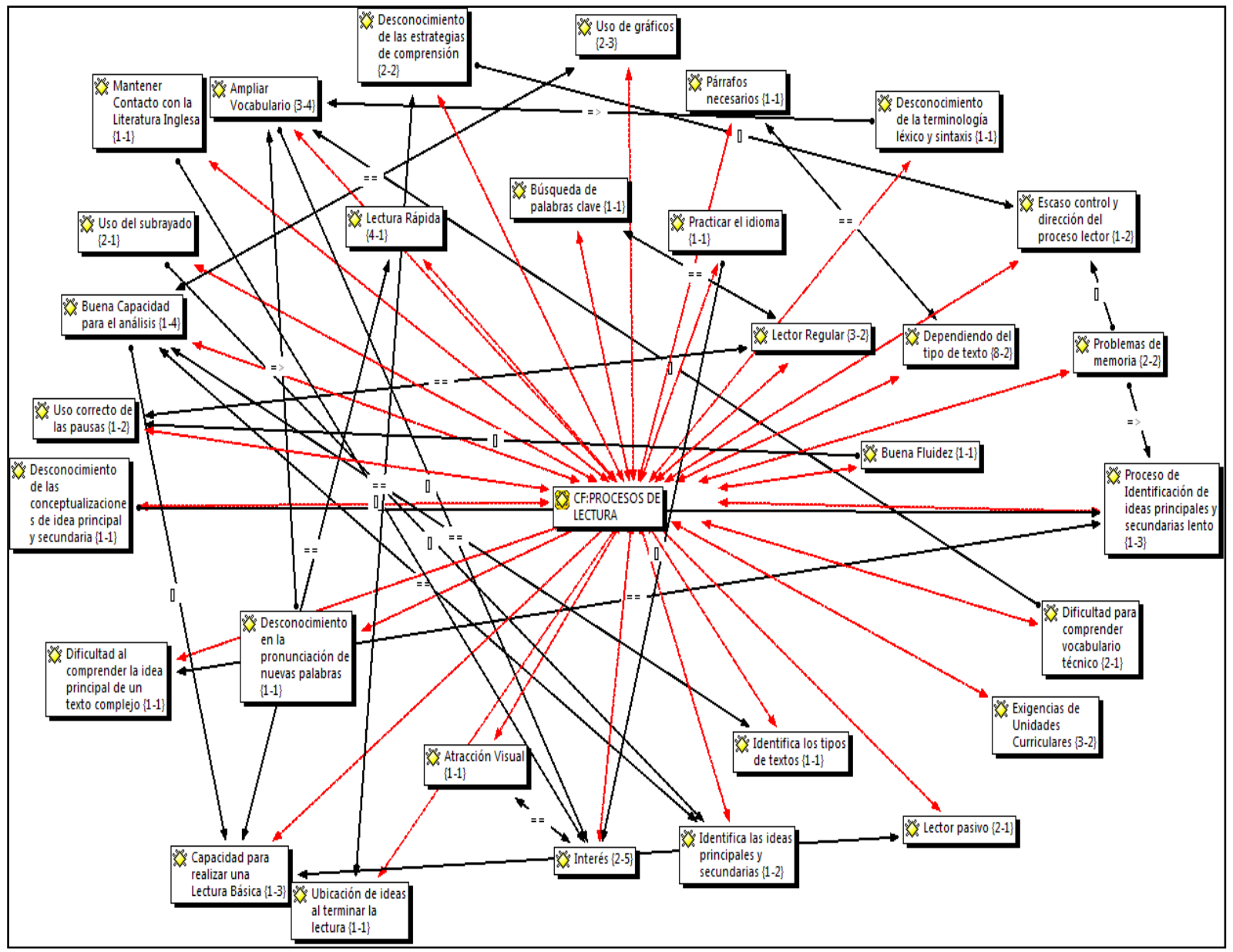

Fuente: Informantes Clave. 
Res., Soc. Dev. 2019; 8(11):e308111474

ISSN 2525-3409 | DOI: http://dx.doi.org/10.33448/rsd-v8i11.1474

El gráfico anterior muestra las relaciones establecidas entre los códigos atendiendo a lo establecido en el Cuadro 1, presentado previamente. La síntesis interpretativa de esta categoría expresa las particularidades evidenciadas en cuanto a las percepciones de los estudiantes sobre los procesos de lectura del idioma inglés.

\section{Categoría $\mathrm{N}^{\circ} 02$ PROCESOS DE ESCRITURA}

Esta categoría representa todo los procesos y elementos que involucra la escritura. En esta red se generaron veintidós (22) códigos, los cuales se interrelacionaron entre sí (ver gráfico 2). De acuerdo a las entrevistas realizadas, uno de los informantes manifestó sentir angustia por el uso incorrecto de la gramática generando errores de sintaxis, esto se debe a que el estudiante presenta escasez de conocimientos previos, es importante recalcar que para realizar un buen escrito es necesario contar con una buena base de la gramática, así como se generó en la categoría $\mathrm{N}^{\circ} 01$ los informantes relataron que también escriben por exigencia de unidades curriculares donde para la elaboración del mensaje estos piensan que deben usar el vocabulario que ya se tiene con el propósito de no obstruir la coherencia en el escrito para en el mayor de los casos evitar errores gramaticales.

Así mismo refieren que hacen uso de gráficos, así como elaborar borradores para saber de qué manera plasmar las ideas. Por otra parte, relatan que existe un rechazo a la escritura debido a la pobreza de vocabulario que presentan, lo que constituye un factor importante ya que el desarrollo de esta habilidad es un requisito fundamental del programa de estudio.

Otra área prioritaria es No poseer un amplio vocabulario, esto representa una constante para quien decide hacer uso del arte escrito. Pues, restringe la autonomía que éste puede tener para desarrollar sus ideas. Sin embargo, cuando esta limitante interfiere en el proceso, los mismos informantes afirman que hacen uso del diccionario o acuden a algún experto para solventar el problema, por lo que esto no impide el gusto por escribir que estos presentan.

También se presenta el uso incorrecto de los signos de puntuación, en conjunto con la omisión de palabras lo que hace que el escrito pierda su esencia, que es transmitir un mensaje. Esto quizás se deba a las metodologías de enseñanza en estudios anteriores o a la no revisión de los reportes o actividades realizadas por el estudiante, dejando que éste se quede con la duda hasta que se topa con la realidad. Si bien es cierto que lo que se plasma en un papel requiere de una escritura clara o lo que es lo mismo que poseer una buena ortografía, 
también es cierto que hacer uso extenso de vocabulario no te hace mejor escritor(a), hacer en esto en demasía y que no tenga la consistencia que se exige para presentar un comunicado sería como presentar letra muerta.

Finalmente, los informantes describen que la revisión es necesaria al momento de entregar el producto final, pues te hace verificar detalles, o agregar información extra que se considere pertinente para hacer el texto consistente.

A continuación, el cuadro 4 muestras las ideas más relevantes expresadas por los estudiantes durante la entrevista correspondiente a esta categoría de análisis.

\section{Cuadro 4 - Categoría. Procesos de Escritura}

\section{Informante Unidad de Análisis}

"Ehh si es más difícil para mí escribirlo, creo que al dejar de escribirlo

1 se pierde un poco ehh la gramática de inglés pero creo que soy más de... de hablarlo un poco"

"Bueno bien, porque no tengo ni letra fea ni bonita, bonita pues, para que no me echen broma me siento bien porque escribo lo que yo quiero y se entiende"

3 "La verdad no me gusta, yo odio la parte escrita pero tengo que mejorarlo" "Me gusta, pero no es algo que me fascina, a veces lo hago porque tengo que hacerlo y en otras me encuentro inspirado y me dejo llevar y lo hago de maravilla. También depende del tipo de texto en general, si quiero hablar de... si va a ser un resumen, una crítica, trato de usar un lenguaje que se pueda entender."

"Bueno esa pregunta es bastante curiosa este porque ehh creo que una vez llegué a escuchar de que cuando una persona comienza a escribir o a expresarse en otro lenguaje o en otro idioma se convierte en una persona totalmente distinta entonces el escribir en inglés no es como si lo estuvieses haciendo en español es como siendo otra persona"

"Bueno me gusta escribir en inglés, trato de por lo menos escribir bastante, ehh trato de ser cuidadosa con la gramática pero si... trabajo con el vocabulario que ya tengo porque si me pongo a escribir cosas que desconozco entonces eso después me va a generar una mala escritura y no es lo que quiero"

Fuente: Los informantes clave.

Los testimonios reflejan los significados atribuidos por los estudiantes al proceso de 
Res., Soc. Dev. 2019; 8(11):e308111474

ISSN 2525-3409 | DOI: http://dx.doi.org/10.33448/rsd-v8i11.1474

escritura del idioma inglés, y avalan la interpretación expuesta a través de la relación de los códigos emergentes. Por otro lado, mostramos en el Gráfico 2, la red semántica correspondiente al análisis de esta categoría.

\section{Gráfico 2 - Proceso de Escritura}

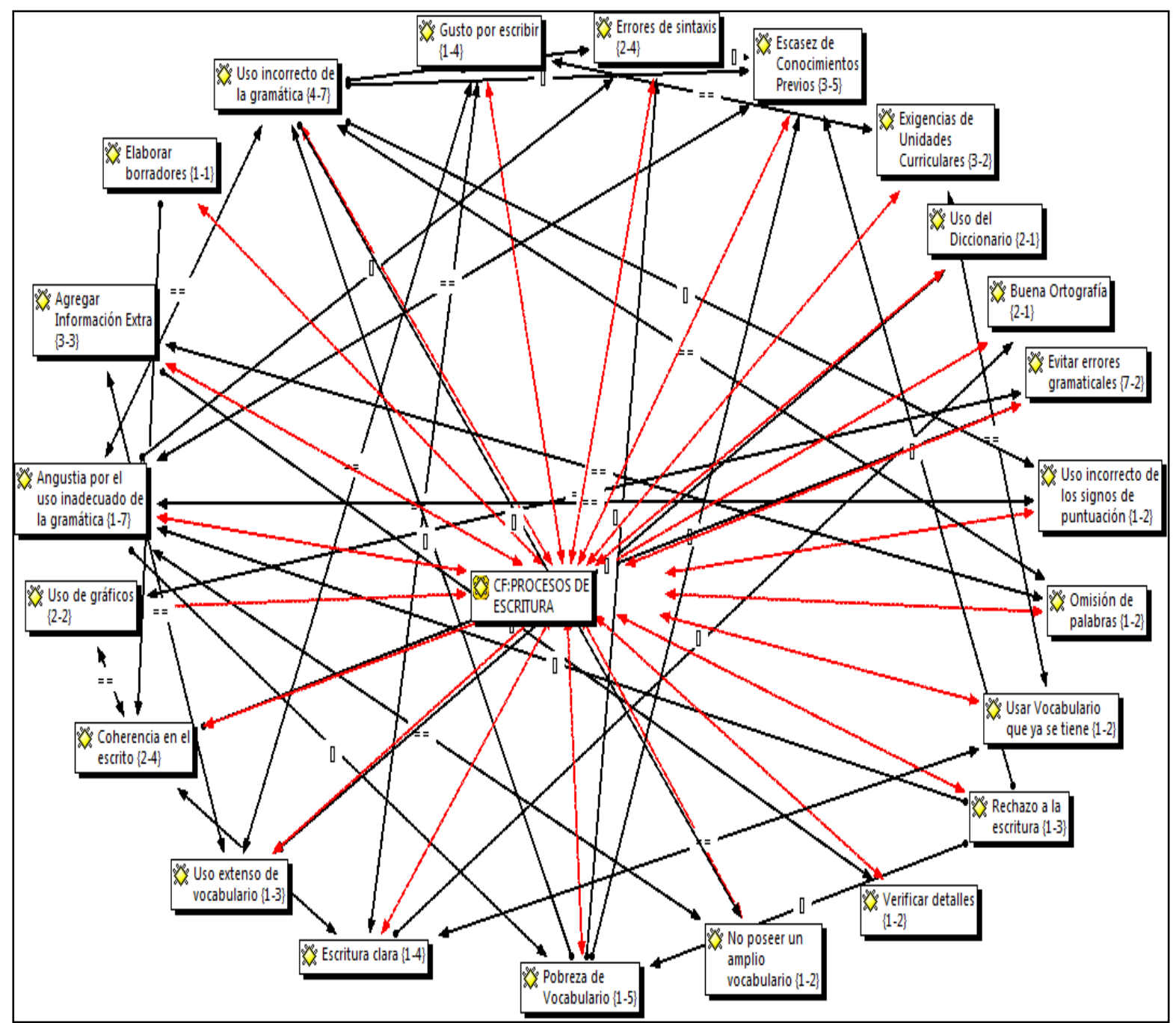

Fuente: Informantes Clave.

Las relaciones correspondientes a la categoría de Procesos de Escritura han sido plasmadas de la red semántica del Gráfico 2, se muestra una visión más completa de os códigos y las interrelaciones que surgen entre sí. 
Para finalizar con la interpretación de las redes semánticas, se tiene la categoría emergente de Necesidades, Gustos e Intereses. Tal y como se expresó anteriormente dicha categoría no se encontraba contemplada en los subtemas de la entrevista en profundidad aplicada. Sin embargo, surgió debido a la variedad en las respuestas de los informantes clave. En este sentido, esta categoría se conforma de trece (13) códigos. Aquí se reflejan los sentimientos, el requerimiento y el provecho que pueda tener la puesta en práctica de la lectura y escritura.

A esto, los informantes clave refirieron que el gusto por escribir lo realizan dependiendo del estado de ánimo, mientras que el placer de leer lo hacen dependiendo del tipo de texto. Cabe destacar que ambos elementos tienen un gran peso en lo que respecta a la comprensión del texto y la escritura, así mismo van de la mano el uno con el otro. Ambos procesos son complejos, hablando en el plano de la escritura, ésta busca que quien escribe sea consciente de la funcionalidad con la que realiza el escrito. Por tanto, involucra sentimientos y emociones al hacerlo. Escribir no se basa sólo en plasmar lo que se piensa, sino saber comunicar, de modo que otros entiendan. Por ello, al hacerlo los informantes sostienen que para no cometer errores en el escrito bien sea de sintaxis o de semántica lo primero que hacen es acudir a algún profesor convirtiéndose esto en una necesidad llámese de este modo al acto de ser vital a algo y no a una carencia. En pocas palabras, es buscar ayuda cuando la necesiten.

Por su parte, para que la lectura sea placentera y eficaz se necesita que quien lee se encuentre enfocado y relajado, pero sobre todo que mantenga una actitud dispuesta hacia el texto. Atendiendo a estas consideraciones, los informantes manifiestan que el placer de leer, así como el de escribir involucra sentimientos y emociones que sólo puede manifestarlo aquel que se encuentra cara a cara con el trozo de papel. Para escribir bien hay que documentarse lo que significa buscar en libros, no sólo en aquellos por los que se tenga atracción visual, pues ya se sabe que no se debe juzgar a un libro por su portada. Además de los libros, pueden usar la internet $\mathrm{u}$ otra herramienta que permita verificar detalles o esclarecer algunas dudas planteadas.

A continuación, presentamos en el Cuadro 5 parte de los testimonios expresados por los estudiantes en esta categoría emergente.

\section{Cuadro 5.}




\section{Categoría. Necesidades, Gustos e Intereses}

\section{Informante Unidad de Análisis}

1 "que sea algo de mi interés, verdad y sobre todo la portada del libro," "para lograr comprender un texto es interpretarlo, debes interpretarlo pues

2 meterte de lleno en la lectura y que nadie te distraiga porque sino no vas a poder y más en el caso del inglés porque no es tu lenguaje propio" "Me gusta, pero no es algo que me fascina, a veces lo hago porque tengo que hacerlo y en otras me encuentro inspirado y me dejo llevar y lo hago

3 de maravilla. También depende del tipo de texto en general, si quiero hablar de... si va a ser un resumen, una crítica, trato de usar un lenguaje que se pueda entender."

..."a través de eso comienzo a desarrollar las ideas que quiero expresar escribiendo..esteee creo que eso viene siendo ya un proceso en el que

4 involucro ehh pensamientos, la imaginación, la creatividad cosas que son importantes este para poder desarrollar aquello que queremos escribir en un texto".

5 "yo odio la parte escrita pero tengo que mejorarlo"

6 "Bueno me gusta escribir y leer en inglés"

Fuente: Informantes Clave.

Evidenciados los testimonios de los informantes, presentamos en el Gráfico 3, la red semántica que define la organización interpretativa de esta categoría emergente. 
ISSN 2525-3409 | DOI: http://dx.doi.org/10.33448/rsd-v8i11.1474

\section{Gráfico 3 - Necesidades, Gustos e Intereses}

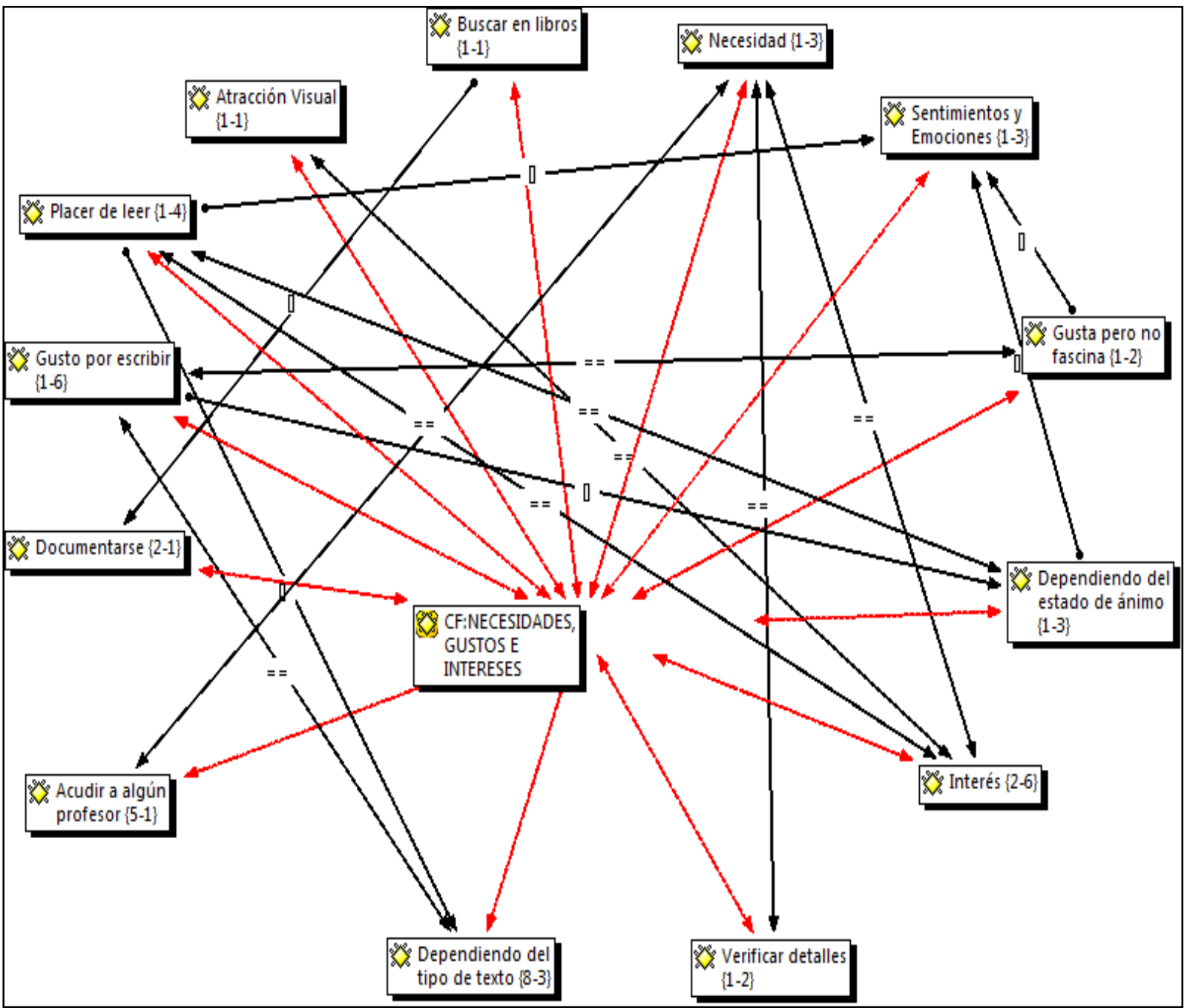

Fuente: Informantes clave.

Sin duda que la categoría emergente conforma el complemento de la realidad evidenciada en cuanto a los procesos de lectura y escritura del idioma inglés de los estudiantes de la UNEFM Municipalizada Carirubana. El Gráfico 3 demuestra que las relaciones establecidas entre los códigos de estas unidades de análisis permiten idearse futuras soluciones a las debilidades evidenciadas, si se toma en cuenta los gustos y preferencias de los mismos estudiantes.

\section{Primeras Aproximaciones}

La interpretación de la información ha permitido realizar una contrastación entre las diferentes teorías que soportan el proceso de lectura y escritura con la exigencia del Sistema 
Educativo Universitario. En este sentido, se detalla cada elemento representado en las redes. Los descubrimientos realizados se ubican en tres (03) categorías, la primera tiene que ver con los procesos de lectura, de acuerdo con los resultados evidenciados se puede describir que, aunque los estudiantes sientan inclinación hacia la lectura, estos carecen de vocabulario, lo que conlleva a que exista una cierta delimitación para realizar la respectiva decodificación.

Aunque la lectura luzca como un proceso fácil por el simple hecho de tomar y leer el escrito, esto no resulta así. Como ya se ha dicho en oportunidades anteriores, es una fase compleja pues requiere que el lector pueda reconocer la información, relacionarla con la que ya se tiene, activar los significados semánticos, que establezca las relaciones pertinentes entre las partes que enlazan el texto, así como la construcción de significados de forma global.

No es un proceso sencillo, pero si se buscan las vías para hacer que todo lo expuesto anteriormente pueda resultar significativo, entonces será de provecho.

Por otra parte, la categoría asignada a los procesos de escritura no ha resultado ser una habilidad muy llamativa para los estudiantes de ILE, de acuerdo con las entrevistas realizadas estos afirmaron que no es algo que les llame la atención, sin embargo, lo hacen por ser este un requerimiento de las unidades curriculares del Programa, así mismo, constataron que prefieren más hablarlo que escribirlo. En efecto, en la interrelación de los códigos se pudo observar que una de las causas que atribuyen a esta problemática es la escasez de conocimientos previos y la ausencia de vocabulario. Para poder elaborar un escrito, este debe seguir una serie de criterios en los que se involucran la cohesión y la coherencia además de muchos otros, la idea es que el estudiante obtenga un nivel de madurez no sólo cognitivo, y analítico sino también lingüístico para que pueda alcanzar los objetivos planteados.

Por otro lado, la parte afectiva y emocional también incide considerablemente en ambos procesos, esto se pudo corroborar en el análisis efectuado a la red semántica que arrojó esta categoría emergente, los estudiantes piensan que los objetivos de la lectura y escritura pueden ser logrados dependiendo del texto que les otorguen y el tipo de estilo en el que escribirán, lo que quiere decir que mantienen una actitud predispuesta ante tales elementos. De ser así esto, los resultados a obtener no serán favorables, ya que ven insignificante ambas actividades. A pesar que ratifican que una tiene más peso que la otra, consideran que de estas se puede obtener un gran beneficio, que sería ampliar el vocabulario, una de los pilares fundamentales para alcanzar las dos competencias mencionadas anteriormente, ya que, a través de la literatura, el lector se topa con nuevo vocabulario el cual pone en práctica la habilidad receptora para luego aplicar la productora (escritura).

Finalmente, resulta importante acotar que para evitar las dificultades que se presentan 
entre estas áreas de aprendizaje, debe existir un enlace en la triada docente-estrategiascontexto real, pues debe existir antes una formación profesional donde el profesor antes de enseñar a otros a leer y escribir éste sea un modelo a seguir, empoderándose de las técnicas que estas necesitan para lograr aprendizajes fructíferos, además de buscar las herramientas necesarias que le permitan al lector/escritor profundizar más en lo que hace, no sólo de interpretar sino de reflexionar y criticar y de forjar nuevos hábitos que desarrollen la plasticidad cognitiva. Y, por último, pero no menos importante introducir variedad de recursos con el propósito de mantener a los estudiantes en contacto con la diversidad de códigos escritos adaptados a las necesidades de cada uno y a la realidad que viven.

\section{References}

Albarrán, J., Albarrán, S., Arenas, L., \& Moreno, D. (2016) El proceso de comprensión lectora en lengua inglesa de los estudiantes de ingeniería: la web quest como herramienta para aprender a leer. ARJÉ. Revista de Postgrado FaCE-UC, 10 (19) 225-239.

Brown, D. (2006). Principles of language learning and teaching. Third edition. Massachusetts: Prentice-Hall Regents.

Díaz-Barriga, F. y Hernández, G. (2004). Estrategias docentes para un aprendizaje significativo. Una interpretación constructivista. Segunda edición. Facultad de Psicología, Universidad Autónoma de México. México: Mc Graw Hill.

Español-López, M. D. L. Á. (2016). Desarrollo del pensamiento crítico a través de la lectura. Moreno, G. C., \& Villafuerte, J. S. (2016). Desarrollo de las destrezas productivas en idioma inglés de estudiantes universitarios. REFCalE: Revista Electrónica Formación y Calidad Educativa. ISSN 1390-9010, 3(3), 89-108.

Oviedo, P. M. (2015). Despertar y encauzar con intención el gusto por la lectura y la escritura. Un imperativo de toda docencia. IE Revista de Investigación Educativa de la REDIECH, 6(11), 7-35

Ramírez, G. C., \& Acosta, J. R. (2015). La enseñanza de lenguas extranjeras: historia, teoría y práctica. Revista de Lenguas Modernas, (22). 
ISSN 2525-3409 | DOI: http://dx.doi.org/10.33448/rsd-v8i11.1474

Yin, R. (1994). Case Study Research. Design and Methods. $2^{\text {nd }}$ ed. London, Thousand Oaks, CS: SAGE.

Zeuch, E. M., \& Gregson, M. (2015). La enseñanza del inglés en la escuela pública venezolana: Evidencias, experiencias y perspectivas. Caracas: British Council/Universidad Central de Venezuela, Facultad de Humanidades y Educación, Comisión de Estudios de Postgrado. Recuperado de http://englishagenda. britishcouncil. org/sites/ec/files F, 145.

\section{Porcentagem de contribuição de cada autor no manuscrito}

Ysamar Sánchez Dávila - 50\%

Jelly Katherine Lugo Bustillos - 50\% 